\title{
C-19
}

\section{POTENCIAL DEL CABLE DE FIBRA ÓPTICA Y DE LA TÉCNICA DTS EN EL RIEGO DE JARDINES}

\author{
Horcajo Diestro, Daniel (1); Rodríguez Sinobas, Leonor (2); Zubelzu Mínguez, Sergio (3); \\ Sánchez Calvo, Raúl (4)
}

1 Alumno, Universidad Politécnica de Madrid, Escuela Técnica Superior de Ingenieros Agrónomos, daniel.horcajo.diestro@gmail.com

2 Profesor, Universidad Politécnica de Madrid, Grupo de Investigación Hidráulica del Riego, Escuela técnica Superior de Ingenieros Agrónomos, leonor.rodriguez.sinobas@upm.es

3 Profesor, Universidad Politécnica de Madrid, Grupo de Investigación Hidráulica del Riego, Escuela técnica Superior de Ingenieros Agrónomos, sergio.zubelzu@upm.es

${ }^{4}$ Profesor, Universidad Politécnica de Madrid, Grupo de Investigación Hidráulica del Riego, Escuela técnica Superior de Ingenieros Agrónomos, raul.sanchez@upm.es

\section{RESUMEN}

El cable de fibra óptica y la técnica Distributed Temperature Sensing puede ser una opción en la programación del riego de jardines para mejorar, no sólo la eficiencia en el uso del agua, sino también, por ende, el de fertilizantes y ahorrar energía. Esta metodología permite estimar en continuo la temperatura hasta $12,5 \mathrm{~cm}$ y temporal de $1 \mathrm{~s}$. Posteriormente, se relaciona la temperatura con el contenido de agua en el suelo obteniéndose errores de hasta $\pm 2 \%$. En este trabajo se aplicó esta técnica al riego por aspersión de un área ajardinada de la ETSI de Agrónomos de Madrid. Los resultados son prometedores dado que permiten conocer la variabilidad espacial de la distribución del agua en el suelo y relacionarla con la distribución del agua por la unidad de riego para elaborar criterios de riego que mejoren la eficiencia de todos los recursos.

\begin{abstract}
The fiber optic cable and the technique Distribute Temperature Sensing show potential to be used for the irrigation programming in green areas, not only to improve the water use efficiency but, the use of fertilizers and saving energy as well. This methodology can measure temperature along the cable, every $12.5 \mathrm{~cm}$, and every second. Then, temperature is correlated with soil water content with up to $\pm 2 \%$ accuracy. This study was performed in a green area located at the ETSI de Agrónomos de Madrid that was irrigated by sprinkler irrigation. The results are promising since the water spatial variability within the soil is known and it can be correlated with the water distribution in the irrigation unit to elaborate better irrigation strategies in the garden.
\end{abstract}

\section{1- Introducción y objetivos}

Los sensores de temperatura distribuida (Distributed Temperature Sensing, DTS) cuentan con un amplio campo de aplicación que incluye, entre otros los campos ambiental 
(Selker et al., 2006a; Selker et al., 2006b; Lowry et al., 2007; Vercauteren et al., 2011; Briggs et al., 2012; 2014; Benitez-Buelga et al., 2014; Benitez-Buelga et al., 2016). Una de sus ventajas es la precisión en la medida de la temperatura de forma continua tanto en el espacio como en el tiempo que se han correlacionado con otras variables como por ejemplo la humedad del suelo $\theta$ (Sayde et al., 2010; Sayde et al. 2014).

EI DTS consta de un láser que emite un pulso luminoso, a una determinada frecuencia, a lo largo del cable de fibra óptica y de filtros y espectrofotómetros. La luz se dispersa en función del índice de refracción de la fibra. La mayor parte de dicha dispersión se produce en la misma longitud de onda que el pulso original, mientras que una porción denominada dispersión de Raman se dispersa a longitudes de onda distintas de las del láser inicial: una parte, denominada Stoke, lo hace a una longitud de onda inferior, mientras que otra porción, llamada anti-Stoke, se dispersa con una longitud de onda superior. La intensidad de la dispersión anti-Stoke es sensible a la temperatura de la fibra, al contrario que la dispersión Stoke. A partir del ratio de la intensidad de las ondas Stokes/Anti-Stokes y del tiempo que tarda la luz reflejada en llegar al punto de emisión, se determina la temperatura en cada a lo largo del cable.

En la estimación de $\theta$, se usa la técnica Active Fiber Optic Temperature System (AFTS) que aplica una potencia calorífica durante un tiempo determinado al encamisado metálico de protección del cable de fibra óptica (Sayde, 2010; Sayde, 2014, Benitez-Buelga et al. 2014).

La mejora en la precisión de la medida depende del cable de FO y del DTS. Como en el resto de sensores para mejorarla, debe existir buen contacto entre el cable y suelo circundante $\mathrm{y}$, además, la instalación debe de calibrarse de forma correcta. Actualmente algunas unidades DTS estiman $\theta$ con una resolución espacial de $12,5 \mathrm{~cm}$ y temporal de $1 \mathrm{~s}$ (Gil et al, 2012; Benitez-Buelga et al., 2016) y con un error de medida pequeño.

El objetivo de este trabajo es estudiar el potencial del cable de fibra óptica y de la técnica DTS para estimar el contenido de agua en el suelo, y su variabilidad, en el riego de áreas verdes.

\section{2- Materiales y métodos}

Para la ejecución de los experimentos se dispusieron de dos instalaciones: por un lado se contó con un recipiente de metacrilato $(2,5 \times 0,5 \times 0,25 \mathrm{~m})$ en el laboratorio del grupo de investigación y en segundo lugar, del área ajardinada "Pico de la Glorieta" de los jardines centrales de la Escuela Técnica Superior de Ingenieros Agrónomos de la Universidad Politécnica de Madrid (ETSIA).

Se tomó muestras de suelo del jardín y se rellenó el recipiente de metacrilato manteniendo la densidad aparente de jardín. Posteriormente, se colocó el cable de fibra óptica tal y como se observa en la figura 1. 


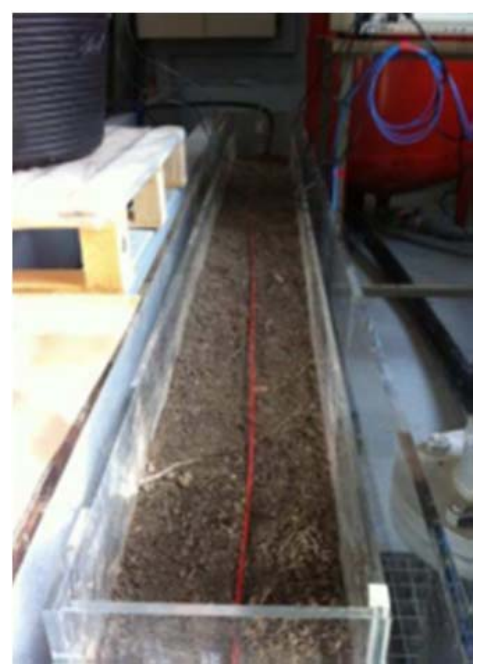

Figura 1. Detalle del contenedor de suelo del jardín con el cable de fibra óptica.

Por su parte, en el jardín se instaló $147 \mathrm{~m}$ cable de fibra óptica enterrados a una profundidad de $0,25 \mathrm{~m}$. La figura 2 muestra un esquema del trazado de la instalación y de la colocación del cable en la zanja.
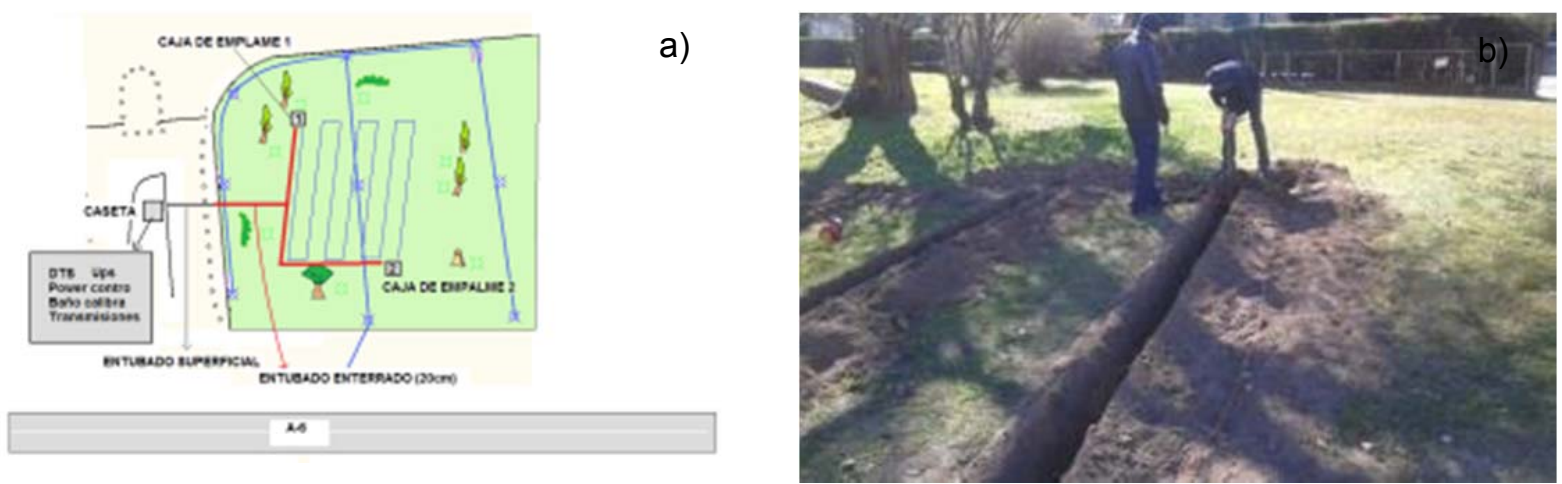

Figura 2. Esquema de la instalación de los jardines de la ETSIA (a) y tendido de la fibra en la zanja (b).

Para la caracterización de la humedad en el suelo $\theta$ se utilizó el concepto de integral térmica $T_{\text {cum }}$ y $\theta$ desarrollado por Sayde et al. (2010):

La medida de la $T_{\text {cum }}$ requiere del uso de la técnica de calentamiento activo de la fibra (Actively Heated Fiber Optics - AHFO -) que consiste en aplicar una diferencia de potencial a la camisa metálica del cable de fibra óptica lo cual requiere de un cable especial que incluya dicha camisa. Para los experimentos se empleó el cable modelo Brusteel de la casa BRUG, la diferencia de potencial al cable se proporcionó mediante un autotransformador modelo Microfusión de marca Control Concepts. Como sensor de temperatura se empleó un DTS modelo Última 004 de la casa comercial Silixa capaz de proporcionar medidas de temperatura cada $0,125 \mathrm{~m}$ y con una frecuencia temporal máxima de $1 \mathrm{~s}$.

Con estas características se calentó la fibra mediante la aplicación de pulsos caloríficos de $7 \mathrm{~W} / \mathrm{m}$ durante $2 \frac{1}{2}$ min con el objeto de obtener una curva de calibración $T_{\text {cum }}{ }^{-}$ $\theta$. Para ello se procedió a saturar el suelo del contenedor y realizar diferentes medidas con diferentes niveles de $\theta$. Para acelerar la perdida de humedad en el suelo se sembró semillas 
de pradera de crecimiento rápido y se aplicó corrientes de aire caliente en el extremo inferior que está perforado. Aparte, $\theta$ se midió con sensores TDR (SM300 Delta-T devices Ltd).

El procedimiento fue el mismo en el laboratorio que en el jardín, realizándose: 5 minutos iniciales, 2,5 minutos de calentamiento y 12,5 minutos finales de enfriamiento. Se programó para registrar una medida de temperatura cada $0,125 \mathrm{~m}$ de cable y cada $5 \mathrm{~s}$ con el procedimiento interno de calibración del DTS y se llevaron a cabo medidas tipo "single ended".

La medida de $\theta$ en el jardín se realizó en dos instantes diferentes: uno para medir el contenido de humedad inicial del suelo y otro, tras aplicar un riego durante $30 \mathrm{~min}$. También se calculó la uniformidad de distribución del agua de riego.

\section{3- Resultados y discusión}

La Figura 3 muestra la curva de calibración $T_{\text {cum }}-\theta$.

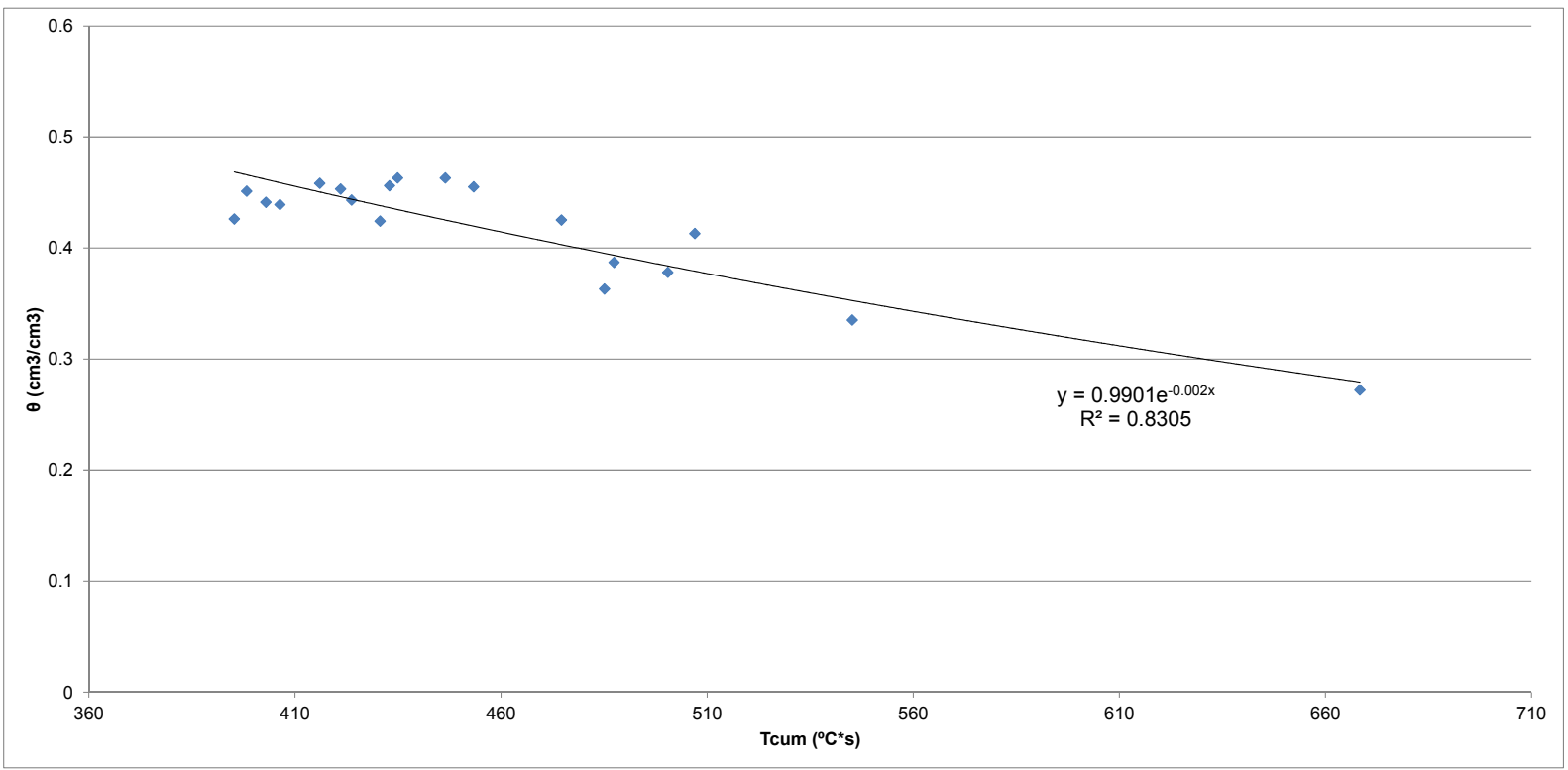

Figura 3. Curva de calibración $T_{\text {cum }}-\theta$.

El mejor ajuste se obtuvo con una curva exponencial con resultados aceptables en cuanto a capacidad explicativa, medida por el coeficiente de determinación $\left(R^{2}\right)$ aunque inferiores a los obtenidos en otros trabajos similares como por ejemplo en Gil-Rodríguez et al. (2011). Este hecho puede deberse a la naturaleza del terreno, formado por rellenos antrópicos recientes que le confieren gran irregularidad al perfil y que dificultad la obtención de y una medida constante de humedad a lo largo del cable de fibra óptica.

Esta curva de calibración se utilizó para estimar $\theta$ en el jardín cuyos resultados se muestran en la figura 4. 


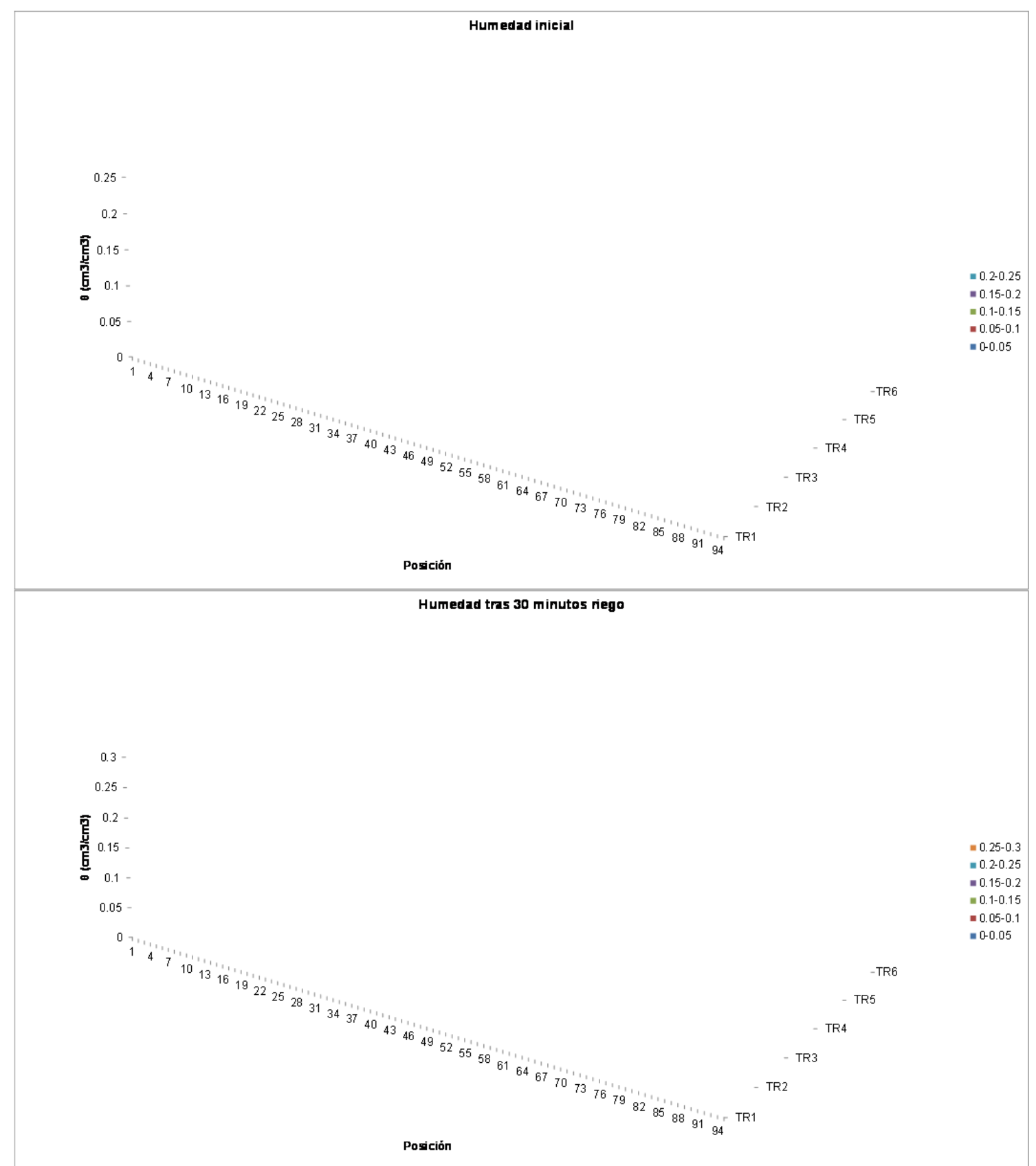

Figura 4. Contenido de agua en el suelo. (a): Antes del riego y (b): tras $30 \mathrm{~min}$ de riego

Los resultados muestran una distribución desigual del agua en el suelo, fruto de un riego con una baja uniformidad $(\mathrm{CV}=0.61)$. Sin embargo, la redistribución del agua mejora parcialmente los resultados de la uniformidad del riego al obtenerse resultados para el coeficiente de variación de $\theta$ de 0,17 y 0,15 correspondientes a las situaciones previa y posterior al riego, respectivamente.

En cualquier caso, la fibra proporciona una herramienta óptima para el control del riego y de la distribución del agua del riego en el suelo, permitiendo un control continuo tanto en el espacio como en el tiempo.

\section{4- Conclusiones y recomendaciones}

El cable de fibra óptica y la técnica del DTS de este trabajo han servido para estimar la variabilidad espacial del agua en el suelo en el riego de jardines. La resolución de medida por parte del aparato es muy alta, permitiendo medir la temperatura cada $12,5 \mathrm{~cm}$, siendo un 
avance en la metrología ya que pueden localizarse zonas que estén con una mayor demanda de agua o que sufran algún cambio (sombreado, raíces, alteración del suelo...).

La sensibilidad y precisión temporal que proporciona el DTS también permiten un avance en el control del riego pudiendo programar de forma precisa los riegos y la lámina de agua aportada al terreno en función de las necesidades.

Hay que tener en cuenta que el cálculo de la humedad del suelo ha sido calculada a partir de la calibración del DTS con un TDR, dando una $R^{2}=0.830$, actualmente se está trabajando para calibrar de mejor manera el aparato pero la problemática aparece en controlar la resolución tan alta que tiene el DTS.

\section{5- Referencias}

Benítez-Buelga, J.; Sayde, C.; Rodríguez-Sinobas, .L., Selker, J., (2014). Heated fiber optic distributed temperature sensing for measuring soil volumetric heat capacity and water content: A dual probe heat-pulse approach. Vadose Zone Journal, 13(11): 13-20.

Benitez-Buelga, J., Rodríguez-Sinobas, L., García-Calvo, R., Gil-Rodríguez, M., Sayde, C., Selker, J., (2016). Calibration of soil moisture sensing with subsurface heated fiber optics using numerical simulation. Water resources research. DOI: 10.1002/2015WR017897.

Briggs, M. A., Lautz, L. K., Mckenzie, J. M., Gordn, R. P., \& Hare, D. K., (2012). Using high resolution distributed temperature sensing to quantify spatial and temporal variability in vertical hyporheic flux. Water resources research, 48.

Gil Rodríguez, M.; Rodríguez Sinobas, L.; Benitez Buelga, J. y Sánchez-Calvo, R.(2013). Application of active heat pulse method with fiber optic temperature sensing for estimation of wetting bulbs and water distribution in drip emitters.. "Agricultural Water Management", v. 120 ; pp. 72-78.

Lowry, C. S., Walker, J. F., Hunt, R. J., \& Anderson, M. P., 2007. Identifying spatial variability of groundwater discharge in wetland stream using a distributed temperature sensor. Water resources research, 43.

Sayde, C., Gregory, C., Rodríguez, M., Tufillaro, N., Tyler, S., Van de Giesen, N., English, M., Cuenca, R., \& Selker, J. S. (2010). Feasibility of soil moisture monitoring with heated fiber optics. Water Resources Research, 46.

Sayde C., Benitez, J. B., Rodríguez, L., Loraine, E. K., English, M., Van de Giesen, N., \& Selker, J. S. (2014). Mapping variability of soil water content and flux across $1-1000 \mathrm{~m}$ scales using the actively heated fiber optic method. Water Resources Research, 50.

Selker, J. S., Van de Giesen, N., Westhoff, M., Luxemburg, W., \& Parlange, M.B..2006a. Fiber optics open window on stream dynamics. Geophysical Research Letters, 33.

Selker, J. S., Thévenaz, L., Huwald, H., Mallet, A., Luxemburg, W., Van de Giesen, N., Stejskal, M:, Zeman, J., Westhoff, M., \& Parlange, M.B., 2006b. Distributed fiber optic temperature sensing for hydrologic systems. Water Resources Research, 42 
AERYDXIV Congreso Nacional de Riegos, Sevilla 2016

Vercauteren, N., Huwald, H., Bou-Zeid, E., Selker, J. S., Lemmin, U., Parlange, M. B., Lunati, I., 2011. Evolution of superficial lake water temperature profile under diurnal radiative forcing. Water resources research, 47. 